\title{
Adaptive Performance Improvement of Fiber Bragg Grating in Radio over Fiber System
}

\author{
Fabrice Mfuamba Kabonzo*, Yunfeng Peng \\ School of Computer and Communication Engineering, University of Science and Technology, Beijing, China \\ Email:"fabriceshao@yahoo.fr, pengf@ustb.edu.cn
}

Received 6 November 2015; accepted 26 February 2016; published 2 March 2016

\begin{abstract}
The combination of Radio Frequency and Optical Fiber has resulted high capacity transmission at lower costs components and makes Radio over Fiber as a current trend of large broadband communication. In Fiber optics field, the use of Fiber Bragg Grating (FBG) was been proposed in recent research with different purpose of uses. However, the compensation of dispersion method of Fiber Bragg Grating (FBG) can boost significantly the system performance. This paper investigates the performance capacity improvement of adaptive Radio over Fiber system. The system design was performed using OptiSystem 7.0 software, which $10 \mathrm{~Gb} / \mathrm{s}$ Non Return to Zero (NRZ) signal was launched into $50 \mathrm{Km}$ Universal Mode Fiber and Fiber Bragg Grating was used as a compensator of dispersion before frequency up conversion. Therefore, the system performances were investigated by comparing the Bit Error Rate (BER) and Q-factors of Positive Intrinsic Negative (PIN) and Ultrafast Avalanche Photodiode (APD) as optical receivers. The Eye diagram analyzer showed acceptable improvement due to use of Fiber Bragg Grating as a compensator of dispersion.
\end{abstract}

\section{Keywords}

Radio over Fiber (RoF), Fiber Bragg Grating (FBG), Dispersion Compensating Fiber (DCF), Positive Intrinsic Negative (PIN), Ultrafast Avalanche Photodiode (APD)

\section{Introduction}

Radio over Fiber is becoming increasingly important for wireless communication in order to support the big data traffic volumes. Currently the integration of Radio Frequency and optical fiber provide enormous bandwidth and reduce significantly the power consumption compared to the others technologies.

Dispersion compensating fiber (DCF) is currently used as the standard solution for dispersion compensation in long distance transmission, since it matched the dispersion cancellation with negligible cascading impairments [6]. However the transmission of light over Dispersion compensating fiber component is limited due to input power to avoid nonlinear impairments that create a high insertion loss over the link. Therefore, Chirped

*Corresponding author. 
Fiber Bragg Grating (FBG) possibly replaces DCF as a standard solution for line dispersion compensation.

Fibers Bragg Grating (FBG) has negligible nonlinearity, low insertion loss and small size sits can possibly impact the system performance and boost the network capacity when used in two different scenarios methods either as a dispersion compensator for long distance fiber network or when used for routing wavelength in Wavelength Division Multiplexing (WDM) systems. In both areas, Fiber Bragg Grating can easily impact the system performance especially when the grating is chirped [1]. The highly selective filtering capabilities of Fiber Bragg Grating combined with its all fiber configuration and flexibility make this technology an ideal candidate for the current and next generation networks [2].

The dispersion compensation of FBG has been demonstrated over $72 \mathrm{Km}$ fiber link leading to error free transmission of $10 \mathrm{G} / \mathrm{bit}$ signal in [3] and also the feasibility of long haul Wavelength Division Multiplexing optical transmission using Fiber Bragg Grating.

This paper investigates the performance improvement of Fiber Bragg Grating in adaptive Radio over Fiber system as considered in [1] and [3]. The scenario solution of long distance link, WDM-RoF system was considered since Radio over Fiber offers lower attenuation loss, better coverage and increased capacity and also is also resistant to Radio Frequency Interferences. The section II discusses the propose work regarding the dispersion compensation using FBG configuration in adaptive Radio over Fiber system.

\section{Proposed System}

The chromatic dispersion is a major issue in the Single Mode Fiber when the signals are transmitted over long distance. The main raison of the proposed system model was to analyze the performance of Fiber Bragg Grating as a compensator of dispersion in Radio over Fiber system. In the system designed, the $10 \mathrm{~Gb} / \mathrm{s}$ Non Return to Zero (NRZ) signal was launched onto $50 \mathrm{Km}$ using Single Mode Fiber (SMF) and the power splitter was used to split the signal into four channels before the frequency up conversion. PRBS (Pseudo Random Bit Sequence) generates the Sequence of Bit Radom. The optical Mach Zehnder Modulator (MZM) was used to modulate the optical source and frequency data together. The continuous wave (CW) was used to provide optical carrier with responsively of $1 \mathrm{~W}$ as illustrated in Figure 2. The optical signal was transmitted over $50 \mathrm{Km}$ Universal single mode fiber and amplified up to $20 \mathrm{~dB}$ due to loss power over long distance transmission. Therefore, at the receiver part the use of power splitter was to split the input signal into four optical signal output. These optical signals are then passed through the optical band pass filters to select the wavelength in frequency of $10 \mathrm{GHz}$.

The use of Fiber Brag Grating in the system proposed in Figure 1 is for compensate the dispersion effect of adaptive Radio over Fiber over long distance transmission.

\section{Simulation and Discussions}

The system designed was performed using OptiSystem software version 7.0. The Figure 2 and Figure 3, demonstrated the performance improvement of Fiber Brag Grating when the line of dispersion was compensated at the Optical receivers. Therefore, at the receivers the results output was validated by analyzed the Q-factor and Bit Error Rate of two different optical detectors with and without using Fiber Bragg Grating device.

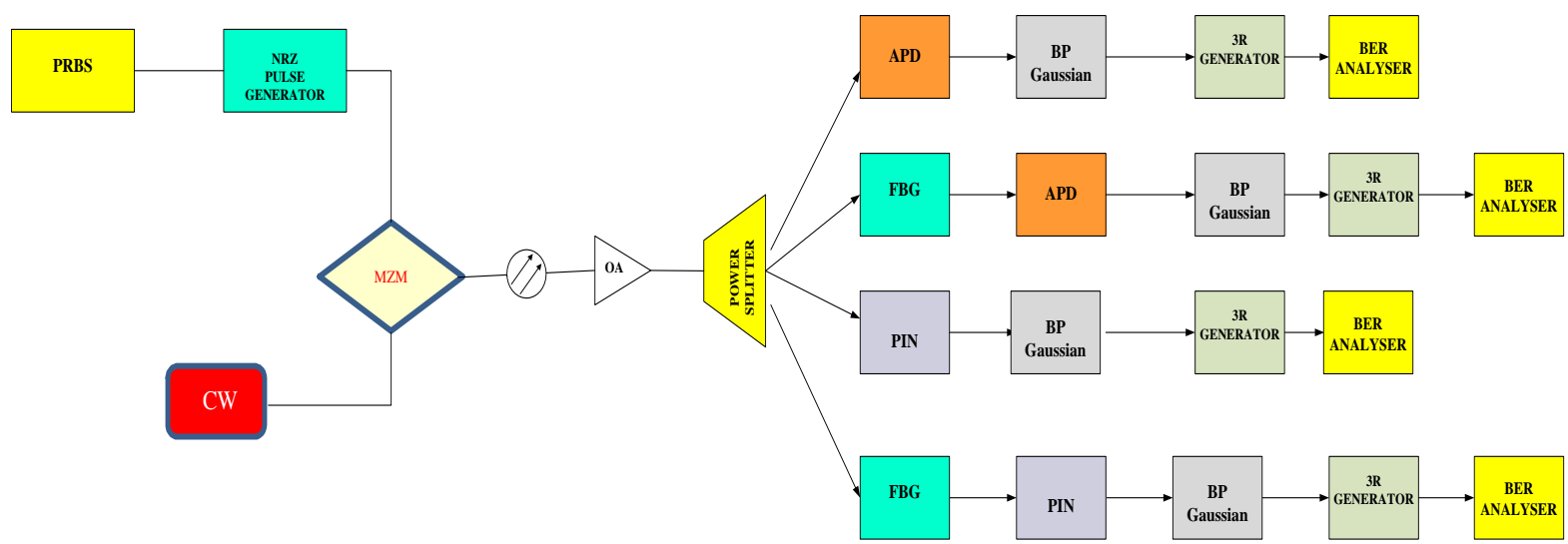

Figure 1. Block diagram of our proposed Adaptive RoF system with FBG. 


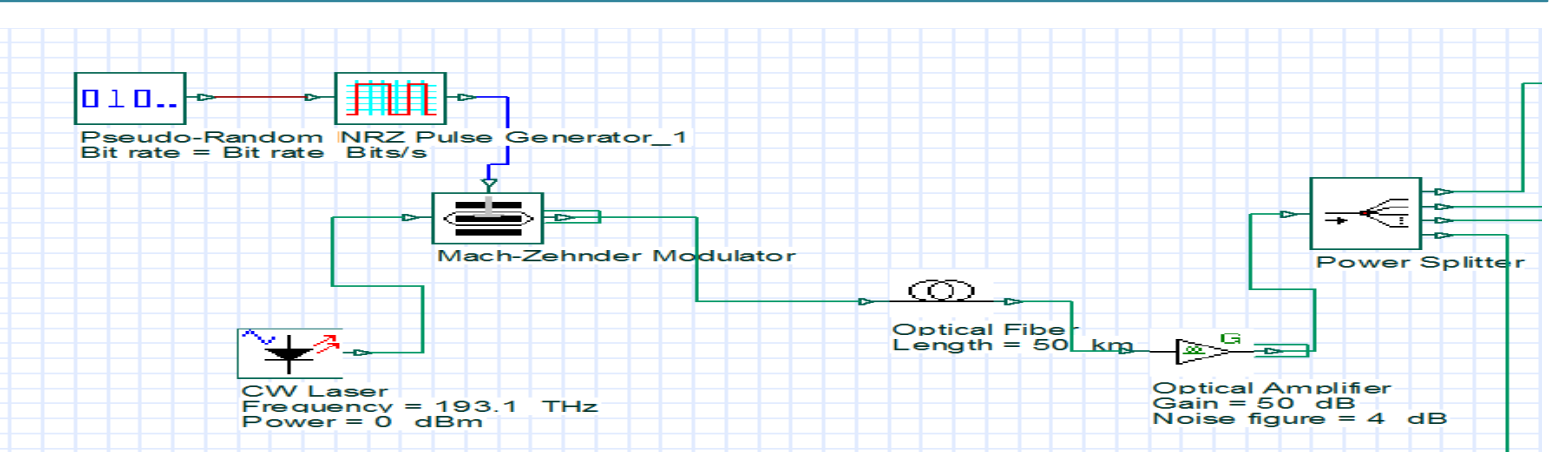

Figure 2. Simulation schematic of RoF transmitter part.

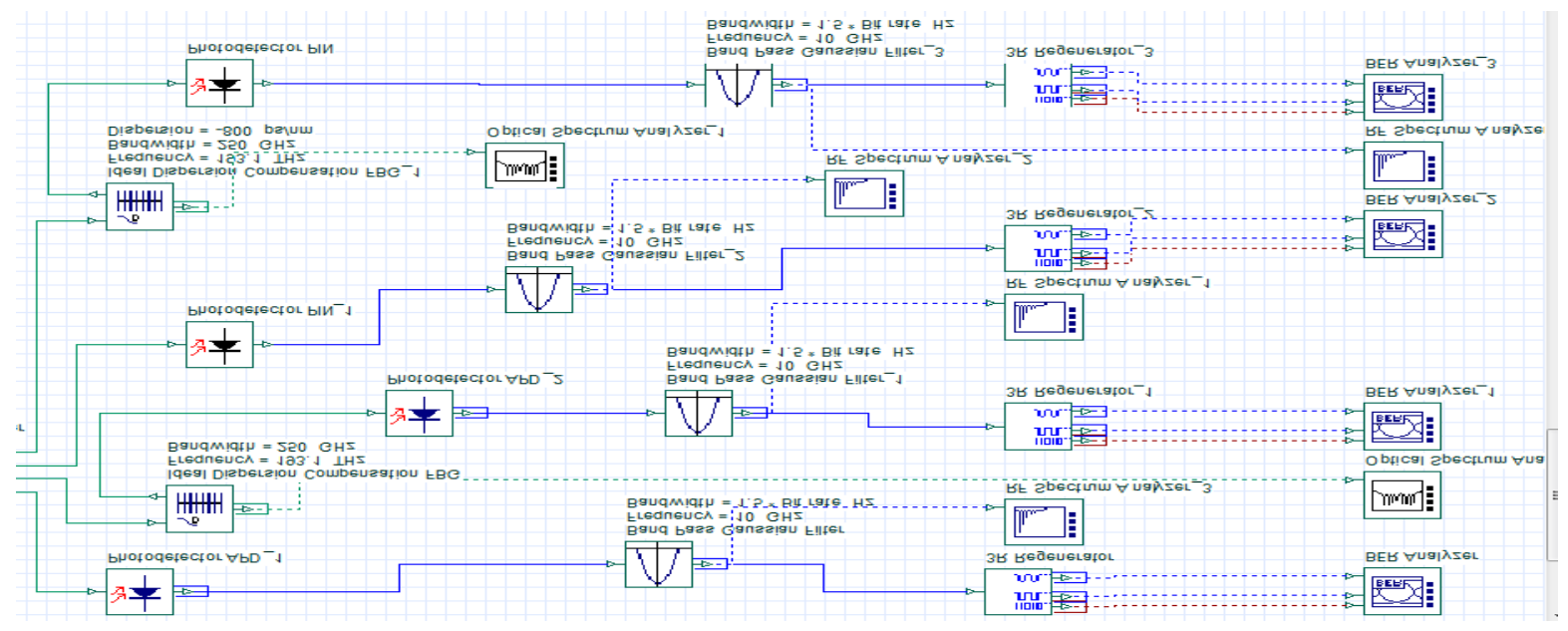

Figure 3. Simulation schematic of RoF receiver part with fiber Bragg grating.

In this paper, the simulation approach was performed in two methods, the first was to employ an Ultrafast Avalanche Photodiode (APD) and the second was to employ Positive Intrinsic Negative (PIN) for comparing the output results of both receivers. The results were evaluated using tree types of analyzers such as optical spectrum analyzer, electrical spectrum analyzer and Bit Error Rate analyzer.

Figure 4(a) presents the results output of system based on the eye diagram analyzer of Electrical signal before employing FBG as compensator of dispersion. Furthermore, Figure 4(b) illustrates the Electrical signal output after compensation made by FBG with PIN as optical receiver. Moreover, Figure 5(a) and Figure 5(b) illustrate the Electrical signals spectrum before and after using Fiber Bragg Grating as compensator of dispersion with APD at the receiver. The results of Ultrafast Avalanche Photodiode (APD) and Positive Intrinsic Negative (PIN) as the receivers are shown in Table 1 and Table 2 respectively. The resulting output of the BER analyzer shows the significant improvement when APD was used with FBG on the channel. Moreover, when PIN was used as receiver, the eye diagram has improves by 5 times as illustrated in Figure 4(a) and Figure 4(b) when the results was compared with and without FBG. Therefore, when APD was used at the receiver, the Q-factor was improved by a factor close to 8 times as illustrated the Figure 5(a) and Figure 5(b) before and after compensation made by FBG. The simulation result shows the acceptable performance improvement of the adapting Radio over Fiber using FBG as a compensator of dispersion.

\section{Conclusion}

In this paper we analyzed the adaptive performance of Fiber Bragg grating as a compensator of dispersion effect over Radio over Fiber system. Here, some electronic components have been eliminated such as the need of electrical modulator and optical demultiplexer has replaced by power splitter, which reduce considerably the cost and complexity of system. In the proposed system, optical signal was directly converted into baseband using only one optical demodulator at the receiver. The performance of the system was evaluated for $10 \mathrm{~Gb} / \mathrm{s}$ using 

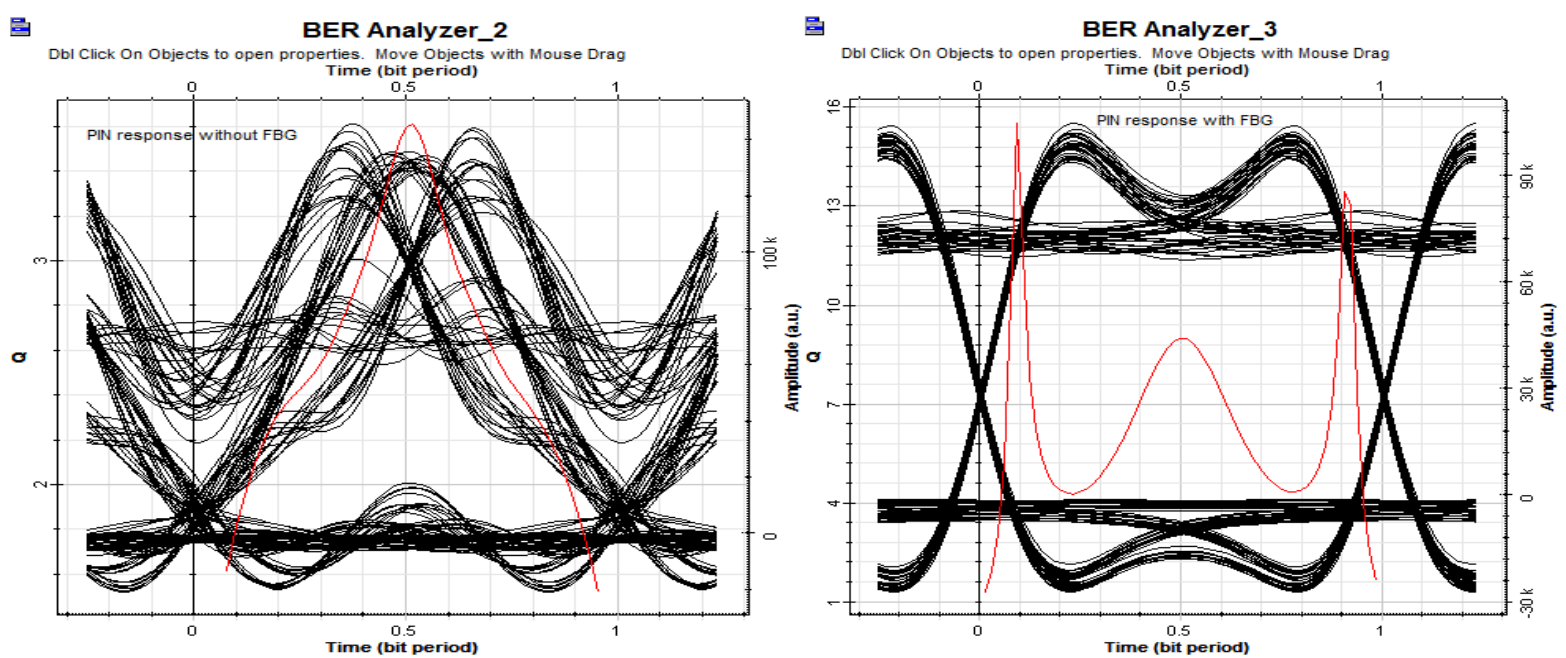

Figure 4. (a) PIN Response before FBG over 50 Km; (b) PIN response after FBG over 50 km.

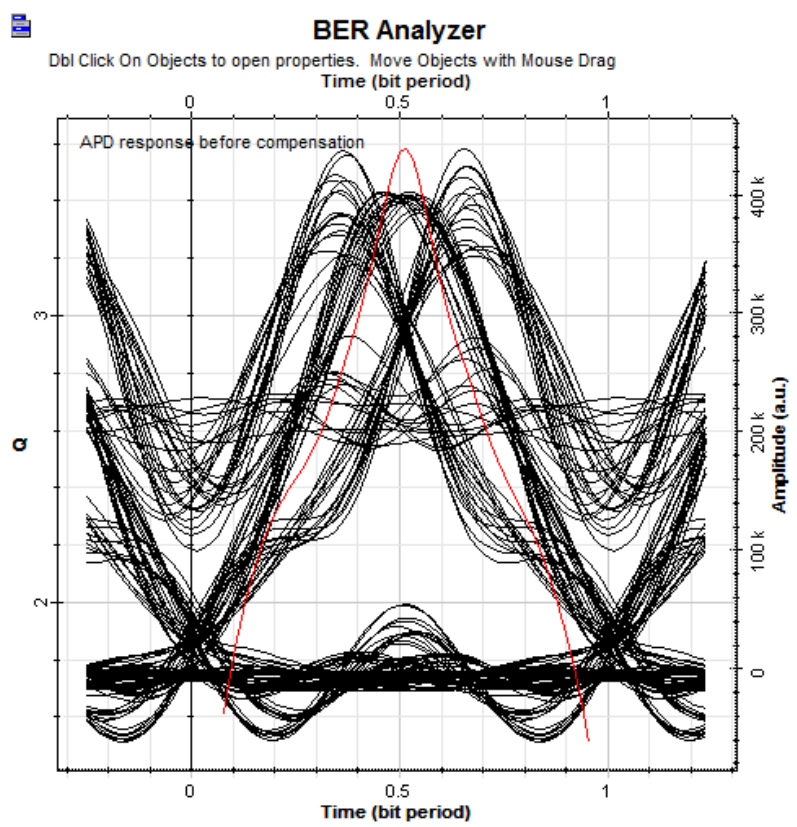

珰

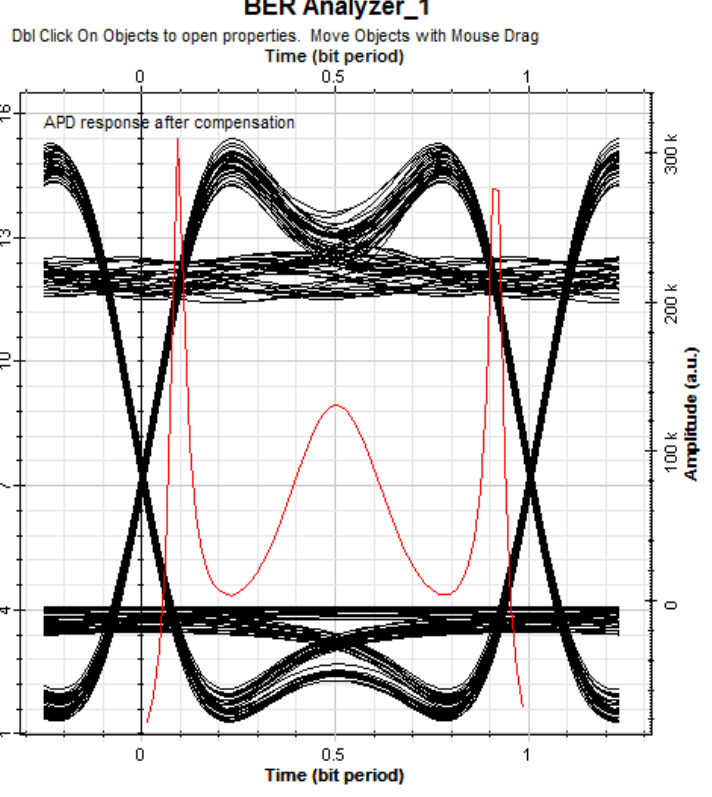

Figure 5. (a) APD Response before FBG over $50 \mathrm{Km}$; (b) APD response after FBG over 50 km.

Table 1. Numerical results APD as receiver.

\begin{tabular}{ccc}
\hline & \multicolumn{2}{c}{ APD Receiver } \\
\cline { 2 - 3 } & Without FBG & With FBG \\
\hline Q-Factor & 3.58158159 & 15.3956 \\
BER & 0.000139952 & $8.7353 \mathrm{e}^{-054}$ \\
\hline
\end{tabular}

Table 2. Numerical results PIN as receiver.

\begin{tabular}{ccc}
\hline & \multicolumn{3}{c}{ PIN Receiver } \\
\cline { 2 - 3 } & Without FBG & With FBG \\
\hline Q-Factor & 3.61174 & 15.5166 \\
BER & 0.000124989 & 1.3385 \\
\hline
\end{tabular}


Fiber Bragg Grating as a compensator of dispersion effect over $50 \mathrm{Km}$ Universal Single Mode Fiber (SMF) of adapting Radio over Fiber System. The Q-factor of the system has been increased by 5 times when an Ultrafast Avalanche Photodiode (APD) is used at the receiver considerably compared to the system using Positive Intrinsic Negative (PIN) as at the receiver. Minimum BER also reduced significantly by using Fiber Bragg Grating, which is shown in Table 1 and Table 2. However, there are still possibilities to extend the technology by discovering a different component for improving the system capacity in the near future.

\section{References}

[1] Arora, O. and Garg, A.K. (2011) Impact of Fiber Bragg Grating as Dispersion Compensator on the Receiver Characteristics. Global Journal of Researches in Engineering Electrical and Electronics Engineering, 11, 19-23.

[2] Kabonzo, F.M. and Peng, Y.F. (2015) Performance Analysis of OFDM Signals in WDM Radio over Fiber System Using Fiber Bragg Grating as a Compensator of Dispersion. International Conference on Information and Communications Technologies (ICT), 1, 85.

[3] Das, S. and Zahir, E. (2014) Performance Evaluation of WDM-ROF System Based on CO-OFDM using Dispersion Compensation Technique. International Journal of Electronics, Electrical and Computational System, 3, 7230-7236.

[4] Kahandawa, G.C., Epaarachchi, J., Lau, K.T. and Canning, J. (2013) Estimation of Strain of Distorted FBG Sensor Spectra Using a Fixed FBG Filter Circuit and an Artificial Neural Network. Centre of Excellence in Engineered Fiber Composites, Vol. 1, IEEE ISSNIP, August.

[5] Li, W., Zhang, X., Zhang, M. and Ye, P.D. (2007) Resolving the Problem of Cross Sensitivity in Fiber Bragg Grating Sensor Based on the Principle of Polarized Light Interference. Frontiers of Electrical and Electronic Engineering in China, 2, 234-239. http://dx.doi.org/10.1007/s11460-007-0043-6

[6] Gayan, C., Jayantha, E., Lau, K.T. and Jhon, C. (2013) Estimation of Strain of Distorted FBG Sensor Spectra Using a Fixed FBG Filter Circuit and an Artificial Neural Network. IEEE ISSNIP.

[7] Litchinitser, N.M., Eggleton, B.J. and Agrawal, G.P. (1998) Dispersion of Cascaded Fiber Gratings in WDM Light wave Systems. Journal of Lightwave Technology, 16, 1523-1529.

[8] Jitender, K., Manisha, B. and Yogendra, S. (2014) Effect of Signal Direct Detection on Sub-Carrier Multiplexed Radio over Fiber System. International Journal of Advanced Research in Computer and Communication Engineering, 3, Issue 1.

[9] Kung, G., Cheng, L. and Xu, M. (2014) Integrated Fiber-Wireless Access Architecture for Mobile Backhaul and Front haul in 5G Wireless Data Networks. Georgia Tech Center for Fiber and Wireless Integration, Georgia Institute of Technology, Atlanta, USA.

[10] Asai, T. (2015) 5G Radio Access Network and its Requirements on Mobile Optical Network. International Conference on Optical Network Design and Modeling, Japan.

[11] Pitakandage, T., Milsavljevic, M., Kourtessis, P. and Senior, J.M. (2014) Cooperative 5G Switched and Adaptive Receiver Beam Forming for Fiber Wireless Networks. 16th International Conference on Transparent Optical Networks (ICTON), Graz, 6-10 July 2014, 1-4.

[12] Ahmed, H., Elias, A. and Maurice, G. (2012) Impairment Aware Radio over Fiber Control Plane for LTE Antenna Backhauling. IEEE ICC-Next Generation Networking Symposium, 2, 2816.

[13] Fahad, A., Khaled, A. and Mohammad, A. (2013) Study of OFDM Technique on RoF Passive Optical Network. Optics and Photonics Journal, June 3, 217-224.

[14] Beas, J. and Garardo, C. (2013) Millimeter Wave Frequency Radio over Fiber Systems: A Survey. IEEE Communication Surveys \& Tutorials, 15, No. 4.

[15] Coelho, D. and Salgado, H.M. (2009) OFDM Signals in WDM Radio over Fiber Networks with Fiber Bragg Grating Selection. ICTON, Page: 1.

[16] Wong, Y.-K., Idruss, S.M. and Ghani, I.A. (2012) Performance Analysis of the OFDM Scheme for Wireless over Fiber Communication Link. International Journal of Computer Theory and Engineering, 4, 807-811.

[17] Wei, C.-C., Lin, C.-T., Huang, H.-T., Liang, W.-L. and Chi, S. (2014) Estimation and Suppression of Dispersion Induced Phase Noise in W-Band Direct Detection OFDM Radio over Fiber System. Journal of Light Wave Technology, 32, 3874-3884.

[18] Almasoudi, F., Alatawi, K. and Matin, M.A. (2013) Study of OFDM Technique on RoF Passive Optical Network. Optical and Photonics Journal, 3, 217-224. http://dx.doi.org/10.4236/opj.2013.32035

[19] Naresh, K., Ami, G. and Sandeep, P. (2012) A Review Radio over Fiber. International of Applied Engineering Research, 7, No. 11. 
[20] Karthikeyan, R. and Prakasam, S. (2013) OFDM Signal Improvement Using Radio over for Wireless Systems. International Journal of Computer Networks and Wireless Communications (IJCNWC), 3, No. 3, June.

[21] Khan, M.I. and Raahemifar, K. (2011) Measurement of Optical Impairments in OFDM Based Radio over Fiber Communication Systems. 21st International Conference on Noise and Fluctuations, Toronto, ON, 12-16 June 2011, 409412. 